\title{
Trabectedin in metastatic soft tissue sarcomas: Role of pretreatment and age
}

\author{
MATHIAS HOICZYK, FLORIAN GRABELLUS, LARS PODLESKA, MARIT AHRENS, \\ BENJAMIN SCHWINDENHAMMER, GEORG TAEGER, CHRISTOPH PÖTTGEN, \\ MARTIN SCHULER and SEBASTIAN BAUER
}

\begin{abstract}
Sarcoma Center, Departments of Medical Oncology, Surgical Oncology and Pathology, West German Cancer Center, University Hospital Essen, University Duisburg-Essen, D-45122 Essen, Germany
\end{abstract}

Received February 7, 2013; Accepted April 2, 2013

DOI: $10.3892 / \mathrm{ijo} .2013 .1928$

\begin{abstract}
Trabectedin has mostly been studied in metastatic leiomyosarcoma and liposarcomas. Only limited data are available in other sarcoma subtypes, heavily pretreated and elderly patients. We retrospectively analyzed 101 consecutive sarcoma patients treated with trabectedin at our center. We recorded progression-free survival (PFS), clinical benefit rate $(\mathrm{CBR}$, defined as complete or partial response or stable disease for at least 6 weeks) and toxicity. Covariates were sarcoma subtype, age and pretreatment. On average, trabectedin was administered for 2nd relapse/progression (range 1st to 12th line). A median of 2 cycles and a dose of $1.5 \mathrm{mg} / \mathrm{m}^{2}$ (range $1-21$ cycles; $1.3-1.5 \mathrm{mg} / \mathrm{m}^{2}$ ) was administered. The median PFS under treatment with trabectedin was 2.1 months in the overall population. Different clinical outcomes were observed with respect to sarcoma subtypes: in patients with L-sarcoma [defined as leiosarcoma and liposarcoma $(n=25)$ ] the CBR was $55 \%$. Notably, long lasting remissions were even observed in 7 th-line treatment. In contrast, the majority of patients with non-L-sarcomas quickly progressed (median PFS 1.6 months). Nevertheless, a CBR of 34\% was achieved, including long-lasting disease stabilization in subtypes such as rhabdomyosarcoma. Patients treated with trabectedin at 1st or 2nd line ( $\mathrm{n}=16)$ achieved an improved PFS (median 5.7 months, range) and a CBR of 59\%. No differences in terms of toxicity or efficacy were observed between patients older than 65 years $(n=23)$ and younger patients $(n=78)$. In this non-trial setting, port-associated complications were more frequent (14\%) with trabectedin compared to other continuous infusion protocols administered at our outpatient therapy center. The majority of patients with relapsing L-sarcomas and a substantial fraction of patients with non-L-sarcomas derive a clinically meaningful benefit from trabectedin. Outpatient treatment is well
\end{abstract}

Correspondence to: Dr Sebastian Bauer, Department of Medical Oncology, West German Cancer Center, University Hospital Essen, Hufelandstr. 55, D-45122 Essen, Germany

E-mail: sebastian.bauer@uk-essen.de

Key words: sarcoma, trabectedin, port complication tolerated also in elderly and heavily pretreated patients. Portassociated complications were observed at an unusually high rate. This suggests a drug-specific local toxicity that merits further investigation.

\section{Introduction}

Soft tissue sarcomas represent a heterogeneous group of mesenchymal tumors. While oncogenic mechanisms vary considerably between different histological subtypes soft tissue sarcomas exhibit similar clinical behaviour with regard to metastatic pattern and sensitivity to classical chemotherapy. High grade sarcomas (G2 and G3 based on the FNCLC classification) show moderate response rates between 20 and $30 \%$ for single drug and 40-60\% for combinational treatments using doxorubicin and ifosfamide (1,2). In 2nd or 3rd line, dacarbacin has been approved based on the data from an early EORTC phase II trial (3).

Trabectedin is a novel chemotherapeutic drug (Ecteinascidin-743, ET743) that was isolated from Ecteinascidia turbinata, a tunicate that grows on carribbean mangrove roots. It is a DNA minor groove alkylator that bends DNA towards the major groove (4) and also interferes with several DNA-binding proteins (5). Trabectedin-binding results in inhibition of gene activation, nucleotide excision repair, induction of DNA strand breaks and cell cycle arrest in S and G2 phases $(6,7)$. The transcription of the MDR1 (multidrug resistance) gene, which encodes for the P-glycoprotein, is suppressed by trabectedin, which appears to explain the lack of cross-resistance with other chemotherapeutic drugs (8).

The single agent clinical activity of trabectedin has been shown in pretreated patients with soft tissue sarcomas in several clinical trials (9-12). While trabectedin rarely induces objective remissions $(<10 \%)$ it shows a substantial progression arrest rate with a favourable toxicity profile which has led to the approval in the European Union for patients with advanced STS after failure of anthracyclines or ifosfamide.

Limited data exist on histological subtypes other than leiomyosarcomas and liposarcomas as well as on patients receiving trabectedin following $>2$ lines of chemotherapy.

To evaluate the activity and feasibility of trabectedin in a routine clinical setting, including older and heavily pretreated 
Table I. Patient characteristics.

\begin{tabular}{|c|c|c|}
\hline \multicolumn{2}{|l|}{ Parameter } & \multirow{2}{*}{$\frac{\%}{100}$} \\
\hline No. of patients & 101 & \\
\hline Median, years (19-83) & 53 & \\
\hline$<40$ years & 19 & 19 \\
\hline $40-60$ years & 50 & 50 \\
\hline$>60$ years & 32 & 31 \\
\hline Male & 55 & 55 \\
\hline Female & 46 & 45 \\
\hline Histology & 101 & 100 \\
\hline Leiomyosarcoma & 24 & 24 \\
\hline Liposarcoma & 22 & 22 \\
\hline Pleomorphic sarcoma & 13 & 13 \\
\hline Synovial sarcoma & 14 & 14 \\
\hline Rhabdomyosarcoma & 6 & 5 \\
\hline $\begin{array}{l}\text { Other (alveolar sarcoma, } \\
\text { chondrosarcoma, desmoplastic } \\
\text { small round-cell tumor, epitheloid } \\
\text { sarcoma, fibromyxoid sarcoma, } \\
\text { MPNST, hemangiopericytoma, } \\
\text { no otherwise specified sarcoma) }\end{array}$ & 22 & 22 \\
\hline \multicolumn{3}{|l|}{ Treatment line } \\
\hline 1st line & 5 & 5 \\
\hline 2nd line & 22 & 22 \\
\hline 3rd or more lines & 74 & 73 \\
\hline
\end{tabular}

Pretreatment included both ifosfamide and doxorubicin; besides gemcitabine and docetaxel were often used; the median age was estimated from date of first trabectedin administration.

patients, we have conducted a retrospective analysis of 101 consecutive patients at the sarcoma clinic of the West German Sarcoma Center.

\section{Patients and methods}

Patients. Between 2001 and 2010, 101 patients with histologically proven diagnosis of locally advanced or metastatic soft tissue sarcomas were treated with trabectedin at the
Department of Medical Oncology of the West German Cancer Center, University Hospital Essen, Germany.

Treatment. Patients received an initial dose of $1.5 \mathrm{mg} / \mathrm{m}^{2}$ over $24 \mathrm{~h}$ as continuous intravenous infusion through a central line (port). Premedication consisted of $20 \mathrm{mg}$ dexamethasone and antiemetic prophylaxis according to our institutional standards. Granulocyte colony-stimulating factor (G-CSF) was not routinely given. Patients had to be fully recovered from any previous treatment-related toxicity (more than grade I CTCAE version 3.0) before administration of the next course. Patients with active viral hepatitis or chronic liver disease or active infection were not treated. Treatment was stopped at clinical or radiological progression, unacceptable toxicity or request by the patient.

Response evaluation and toxicity. All clinical and laboratory examinations were conducted following our institutional standards and were documented in the patient charts. Imaging was performed after the first 2 cycles and subsequently every 6-9 weeks. The progression-free survival (PFS) time was estimated from start of the first cycle of trabectedin until the documented disease progression. Clinical benefit rate was defined as partial remission or radiologically documented (according RECIST) stable disease for at least 6 weeks. Toxicities were extracted from the patients' charts and graded according to the National Health Institute (NHI) common toxicity criteria (CTCAE 3.0).

Statistical analysis. Data are presented as percentage or median plus range unless otherwise specified. Statistical analysis was performed using SPSS 17.0 for Windows (SPSS Inc., Chicago, IL, USA). Survival probabilities were calculated using the Kaplan-Meier method, and differences between the survival curves were assessed using the log-rank test, with p-values $<0.05$ considered statistically significant.

\section{Results}

The population consisted of 101 patients with a median age at time of first trabectedin of 55 years (range 19-83 years; $55 \%$ male $/ 45 \%$ female). All patients had confirmed diagnosis of soft tissue sarcoma and included 24 leiomyosarcomas (LMS), 22 liposarcomas (LPS), 13 pleomorphic sarcomas, 14 synovial sarcomas, 6 rhabdomyosarcomas (RMS), and 22 patients with other sarcoma subtypes (Table I).

Table II. Progression-free rates in different sarcoma subgroups.

\begin{tabular}{lcccc}
\hline & L-sarcoma & Non-L-sarcoma & Elderly & Non-elderly \\
\hline $\mathrm{n}$ & 46 & 55 & 23 & 78 \\
Median PFS & 3.1 & 1.6 & 2.9 & 2.1 \\
3 months PFS & $51 \%$ & $36 \%$ & $42 \%$ & $43 \%$ \\
6 months PFS & $38 \%$ & $16 \%$ & $30 \%$ & $26 \%$ \\
CBR & $55 \%$ & $34 \%$ & $43 \%$ & $44 \%$ \\
\hline
\end{tabular}

$\mathrm{CBR}$, clinical benefit rate $(\mathrm{NC}+\mathrm{PR})$. 


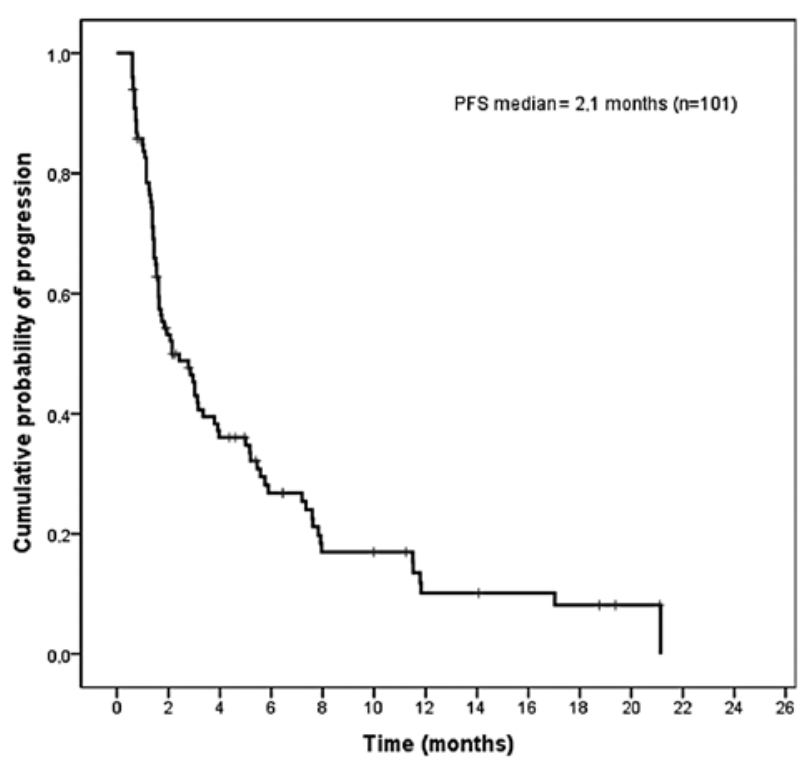

Figure 1. Progression-free survival (months) in all patients calculated from start of trabectedin $(\mathrm{n}=101)$.

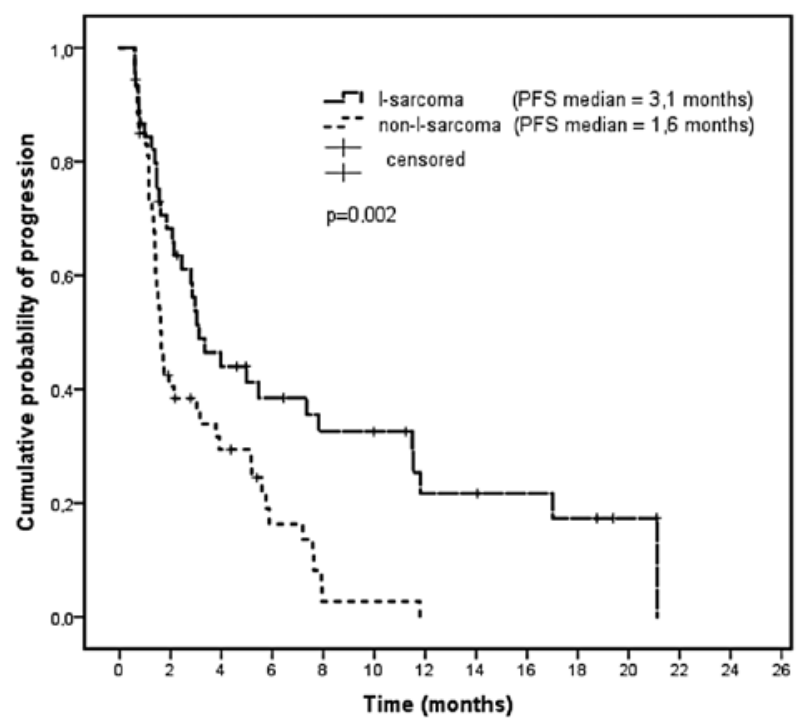

Figure 2. Progression-free survival curves comparing L-sarcomas $(n=46)$ with non-L-sarcomas.

First-line treatment with trabectedin was given in 5 patients, 2nd-line treatment in 22 patients, 3rd-line treatment in 31 patients and 43 patients received trabectedin later than 3rd-line (Table I).

The median PFS of the entire population was 2.1 months (Fig. 1). A median of 2 cycles at a median dose of $1.5 \mathrm{mg} / \mathrm{m}^{2}$ (range 1-21 cycles; $1.3-1.5 \mathrm{mg} / \mathrm{m}^{2}$ ) was administered. L-sarcoma patients received trabectedin on average in 3rd-line, with a median PFS of 3 months and a CBR of 55\% (Fig. 2). This translated into a 3 -month PFS of $51 \%$ and a 6 months PFS of 38. Notably, long lasting remissions were even observed in 7th-line treatment. In this subgroup, a significant difference in PFS (21 vs 3 months) was found in patients receiving

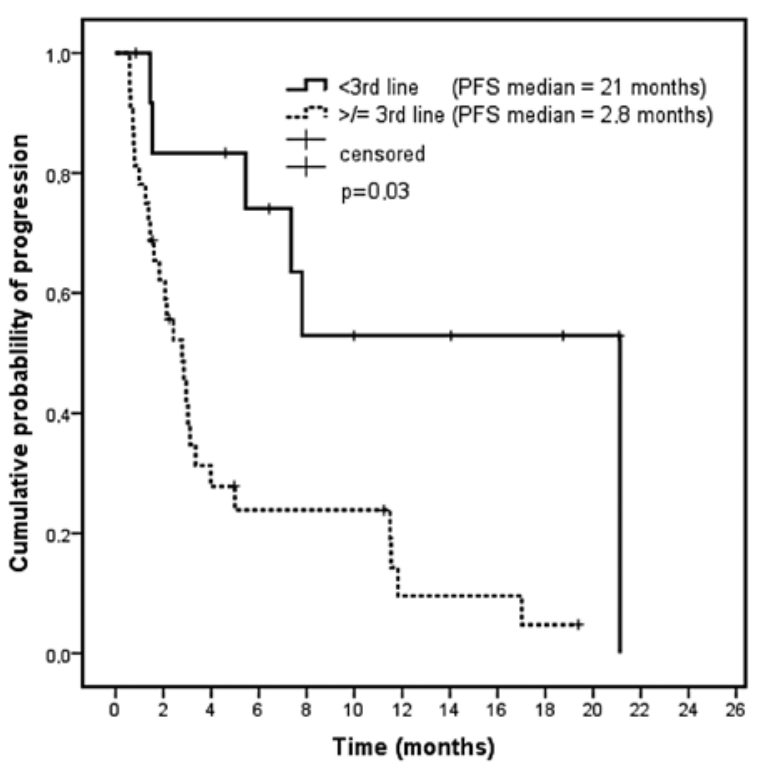

Figure 3. Progression-free survival in L-sarcomas depending on treatment line.

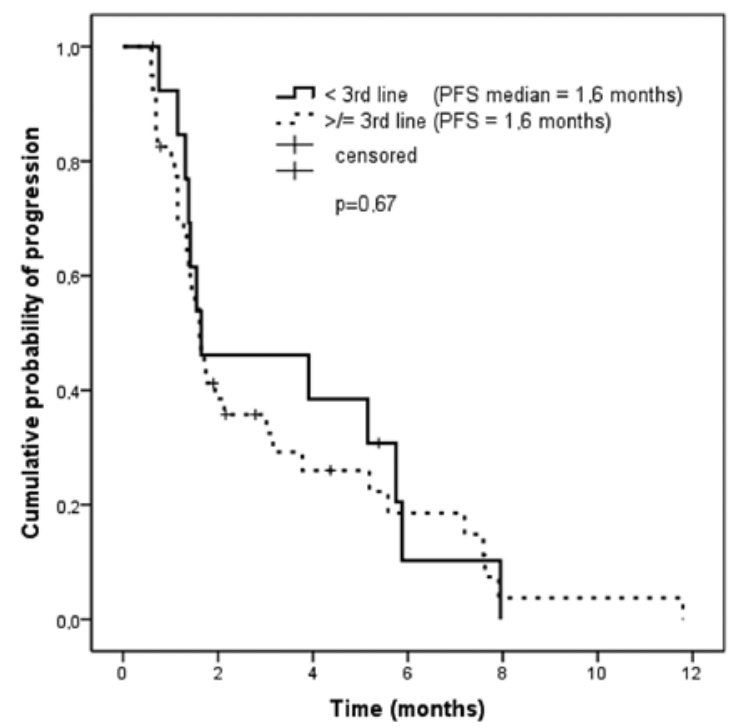

Figure 4. Progression-free survival in non-L-sarcomas depending on treatment line.

trabectedin in 1st/2nd-line treatment versus those treated in 3rd or higher lines ( $\mathrm{p}=0.003$, t-test) (Fig. 3). Partial remissions were only seen in seven L-sarcomas patients. Of note one leioand one liposarcoma patient, who received trabectedin in the seventh treatment line experienced a disease stabilization of 11 and 19 months (Table II).

In patients with non-L-sarcomas CBR was 35\% ( $\mathrm{n}=19)$ with a median PFS of 1.6 months (Fig. 2). Two of seven RMS patients experienced prolonged stabilization of $\leq 8$ months (pleomorphic, 2) despite heavy pretreatment (Fig. 2). We did not observe a difference in progression-free rates when comparing patients who received trabectedin in earlier $(<3$ rd-line $)$ with those who received it in later treatment lines (Fig. 4). 
Table III. Adverse events documented according to common toxicity criteria (CTC).

\begin{tabular}{lcc}
\hline Adverse event & Grade I/II (n) & Grade III/IV (n) \\
\hline Emesis & 40 & 2 \\
Nausea & 75 & 4 \\
Fatigue & 60 & 5 \\
Fever & 20 & 1 \\
Diarrhea & 22 & 2 \\
Constipation & 30 & 1 \\
Acute renal failure & 2 & 0 \\
Port catheter-associated & 9 & 13 \\
complications & & \\
Anemia & 42 & 3 \\
Leukocytopenia & 13 & 5 \\
Thrombocytopenia & 9 & 5 \\
AST/AP elevation & 36 & 4 \\
\hline
\end{tabular}

AST, aspartate aminotransferase; AP, alkaline phosphatase.

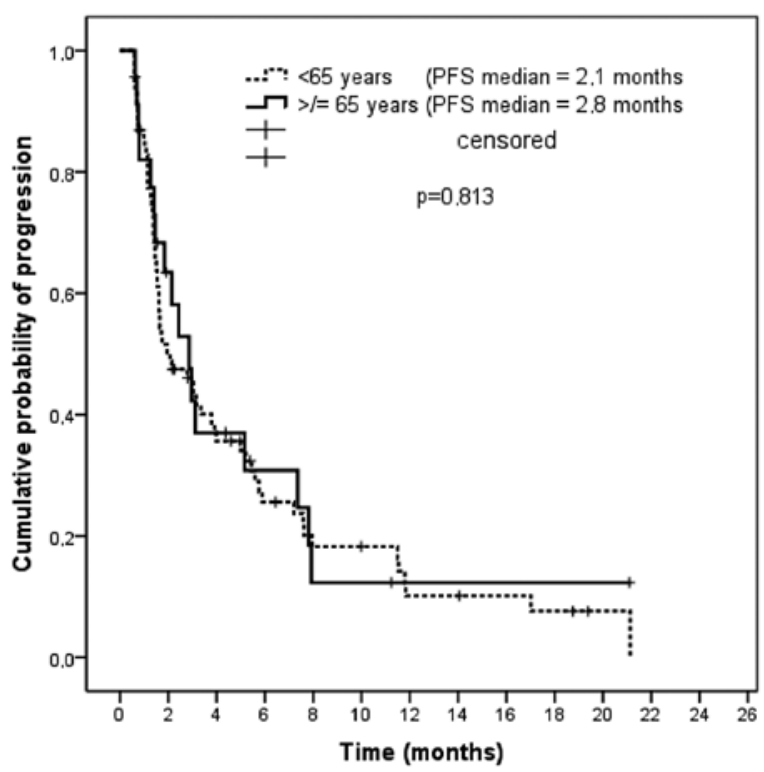

Figure 5. Progression-free survival (in months) in elderly ( $>65$ years) compared to younger patients.

Dose reductions were necessary in 13 patients, mostly due to hematological toxicities (thrombocytopenia $\mathrm{IV}^{\circ}$ ); a dose reduction was performed in one patient receiving trabectedin in 1st line (fatigue grade III), 7 patients in the 2nd line (fatigue grade II, neutropenia grade IV; thrombocytopenia grade III), 3 patients in 3rd line (irritation of port-catheter site; creatinine kinase elevation grade III), 1 patient in the 4 th and 6th line each. While toxicities affecting dose intensity did not appear to be more common in pretreated patients, mild hematological toxicities occurred more frequently (Table III).

In the elderly population ( $>65$ years; $n=23$ ) trabectedin was given in 3rd line (median) and was well tolerated. Dose reductions were necessary in 3 elderly patients (fatigue CTC grade II and III, leukocytopenia grade IV), no treatment discontinuation was necessary. Seventy-eight percent of all patients received trabectedin in 3rd line or higher (median number of pretreatments, 4 drugs). In these patients, an increased incidence of CTC grade 1-2 hematological toxicities was observed. However, dose reductions were not increased compared to patients receiving trabectedin in 1 st- or 2 nd-line treatment.

In this non-trial setting, port-associated complications (paravasation, irritation, infection) were more frequent $(13 \%)$ than in continuous infusion protocols with other drugs (e.g., 5-FU) administered in our large outpatient center.

\section{Discussion}

Patients with metastatic soft tissue sarcomas are still faced with a dismal outcome. For decades only 3 drugs, doxorubicin, ifosfamide, and dacarbacin, have been used, and the median OS has been 12-14 months in these patients (13). Sarcomas occur at all ages and in clinical practice young patients with good performance status often run out of approved chemotherapeutic options. Elderly patients with poor PS have few systemic treatment options that offer a favorable toxicity profile. Trabectedin has recently been approved for treatment of STS after failure of anthracyclins and ifosfamide and has been shown to be particularly active in L-sarcomas.

Here, we present our experience with trabectedin in the setting of a large academic sarcoma center, including a considerable number of elderly, heavily pretreated patients and patients with sarcomas other than LMS and LPS (53\%).

With regard to clinical activity, we have seen a median PFS of 3 months and a CBR of 54\% in L-sarcomas which compares favorably with published data by Demetri et al and Fayette et al $(14,17)$.

In our cohort patients with L-sarcomas who received trabectedin in 1st or 2nd line had a better PFS than those who were treated in 3rd or higher line ( 21 vs 2.7 months). In this context a large clinical trial is currently investigating the role of trabectedin in comparison with doxorubicin in untreated sarcomas (EORTC 62091; Doxorubicin Hydrochloride or Trabectedin in Treating Patients with Previously Untreated Advanced or Metastatic Soft Tissue Sarcoma). One explanation for this difference could be the disproportionate number of patients with myxoid liposarcomas who were treated in the early treatment line ( $n=4$ in 1 st or 2 nd line). Myxoid liposarcomas appear to be the most sensitive sarcoma subtype (16). Notably long-lasting treatment stabilization was observed even in heavily pretreated patients (7th-line treatment).

In patients with other than lipo- and leiomyosarcoma $58 \%$ of the patients exhibited immediate progression after 2-3 cycles of therapy. This might in part be explained by the fact, that these patients had received trabectedin at higher lines than patients with L-sarcomas (non-L-sarcoma $>4$ th line $26 \%$; L-sarcoma $>4$ th line 19\%). Also in non-L-sarcomas a considerably number of patients showed prolonged disease stabilization, including subtypes not well known to be trabectedin-sensitive, such as rhabdomyosarcomas (embryonal, 2; pleomorph, 5) or synovial sarcomas. Two patients with rhabdomyosarcoma had a progression-free survival of 7 and 8 months. For non-pleo- 


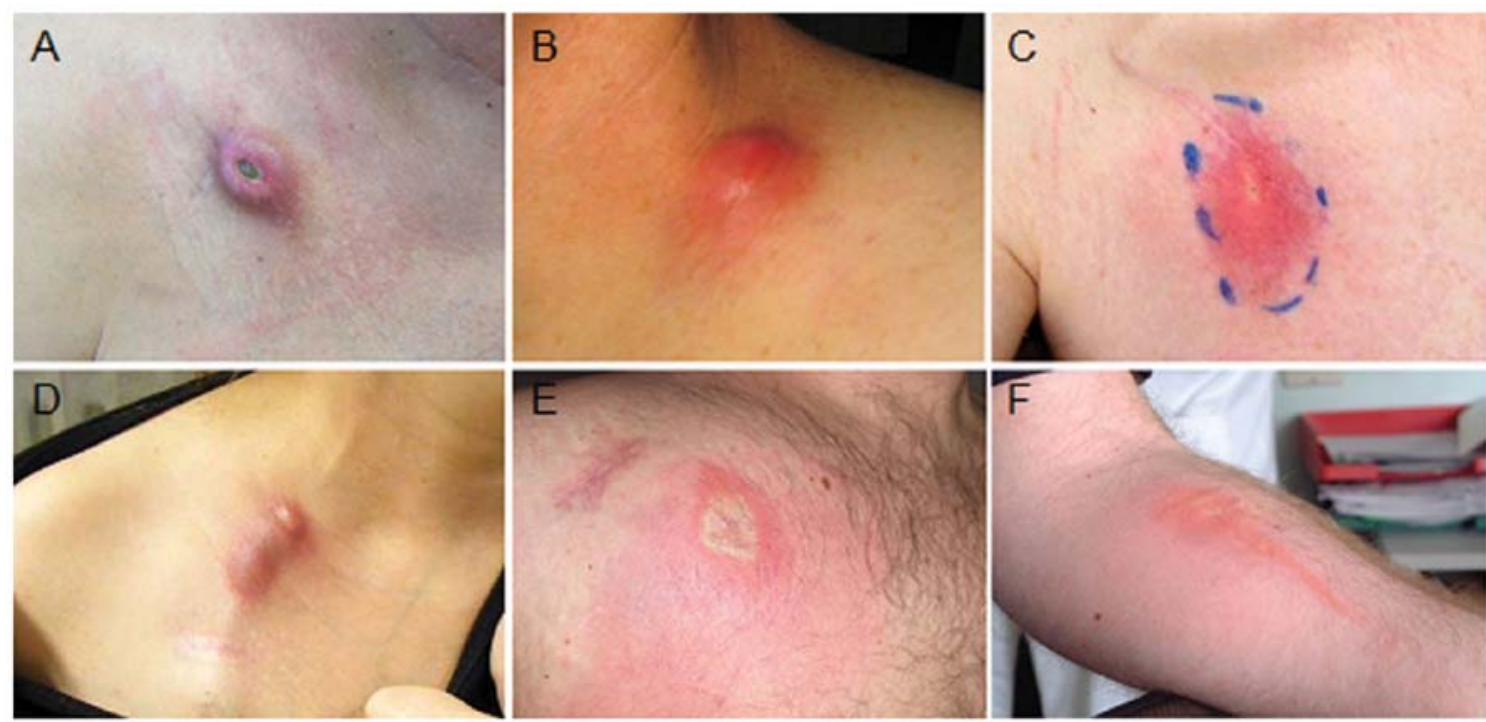

Figure 6. Images of port complication. (A-E) Clinical examples of non-infectious irritation at the port catheter site of the port system. (F) A strong thrombophlebitic reaction after accidental application through a peripheral vein.

morphic rhabdomyosarcomas, a phase II trial of trabectedin in pediatric patients has demonstrated a single partial remission but only very limited disease control rate in a pediatric sarcoma population (15).

For non-L-sarcomas we did not see an advantage of treating patients at earlier treatment lines but again observed many patients who exhibited a clinical benefit despite a substantial number of previous lines of chemotherapy.

Our cohort included 23 patients $>65$ years. Interestingly, we did not observe any difference in PFS when compared with patients $<65$ years (Fig. 5). Also, the use of trabectedin in elderly patients was not associated with decreased dose intensity in our series.

These data underline that trabectedin offers a meaningful alternative in elderly patients in clinical practice. In a similar analysis, Fayette et al (17) reported a higher benefit for patients with good performance status (WHO PS 0-1). In our patients the vast majority of patients was treated in an outpatient setting suggesting a good PS in most of them. However, performance status was not a covariate in our analysis. Nonetheless, we have not observed cumulative toxicity and would not preclude treatment from patients with reduced performance status from a treatment with trabectedin.

Despite the limitations of a retrospective analysis the toxicity profile in our patients was comparable to that described in prospective trials (14). Grade III/IV toxicities were a rare exception and only one patient developed a lifethreatening complication. In this case, a severe pneumonia requiring mechanical ventilation occurred in a 74-year-old patient following one cycle of trabectedin. Neutropenic fever, as described in the clinical trials, was very rarely seen.

A unique side effect that we believe has been underestimated in connection with trabectedin was observed in 14 patients (14\%). A non-infectious irritation of the port site with various degrees of severity was a common finding in our clinical practice (Fig. 6). Similar to the case report by Theman et al (18) a rash occurred around the port system or along the subcutaneous line leaving the metal port. In many cases, the flow of the port system had been reduced, with small thrombi adjacent to the tip of the port line. Bacterial port infections were unlikely as we usually obtained sterile port cultures and patients did not develop fever. However, the irritation usually caused erythema, pain and itching, which was perceived as very uncomfortable by the patients. The erythema usually persisted for several weeks and then spontaneously faded. In patients with associated port catheter occlusion, usually a new system was implanted. Some patients required several consecutive port replacements, which is extremely rare in patients e.g., with colon cancer who receive 5-FU based treatments in our practice.

While a transvasation of the drug through the port-line cannot be excluded, we hypothesize that an increased resistance of the port system caused by small thrombi at the tip of the port line is associated with a backspill of the drug. Other vesicants, such as doxorubicin or vinorelbin, are usually not given as 24-h infusion outside the hospital environment or oncological practice which might reduce the risk of portneedle translocations.

In contrast to the 'toxic-looking' anthracyclins, trabectedin is colorless and paravasations are more difficult to detect both for medical staff as well as for patients. In our experience, a paravasation does not cause immediate irritation or burning sensations. All this may affect the threshold of patients to seek doctor's advice as well as the reaction of medical staff to aggressively treat paravasations.

We believe that patients should be clearly advised about this side effect and the risk of port dislocation. We have changed our practice in that patients whose port systems show increased resistance or a low spontaneous infusion flow undergo radiological contrast-enhanced imaging to detect port-associated thrombi. If treatment with 10,000 IU of streptokinase does not re-establish a good flow port catheters are replaced.

In conclusion, our analysis confirms that $\sim 50 \%$ of patients with L-sarcomas derive clinical benefit from trabectedin 
regardless of the extent of pretreatment in a routine setting. Moreover, significant activity is found in a subgroup of patients with non-L-sarcomas including rhabdomyosarcoma. Administration of trabectedin on an outpatient basis is well tolerated in elderly and heavily pretreated patients. The increased incidence of port complications merits further investigation and should be part of patient and medical staff guidance.

\section{References}

1. Sleijfer S, Ouali M, van Glabbeke M, et al: Prognostic and predictive factors for outcome to first-line ifosfamide-containing chemotherapy for adult patients with advanced soft tissue sarcomas: an exploratory, retrospective analysis on large series from the European Organization for Research and Treatment of Cancer-Soft Tissue and Bone Sarcoma Group (EORTC-STBSG). Eur J Cancer 46: 72-83, 2010.

2. Reichardt P: High-dose chemotherapy in adult soft tissue sarcoma. Crit Rev Oncol Hematol 41: 157-167, 2002.

3. Antman K, Crowley J, Balcerzak SP, et al: An intergroup phase III randomized study of doxorubicin and dacarbazine with or without ifosfamide and mesna in advanced soft tissue and bone sarcomas. J Clin Oncol 11: 1276-1285, 1993.

4. Zewail-Foote M and Hurley LH: Ecteinascidin 743: a minor groove alkylator that bends DNA toward the major groove. J Med Chem 42: 2493-2497, 1999.

5. Minuzzo M, Marchini S, Broggini M, et al: Interference of transcriptional activation by the antineoplastic drug ecteinascidin-743. Proc Natl Acad Sci USA 97: 6780-6784, 2000.

6. Takebayashi Y, Pourquier P,Zimonjic DB, et al: Antiproliferative activity of ecteinascidin 743 is dependent upon transcriptioncoupled nucleotide-excision repair. Nat Med 7: 961-966, 2001.

7. Damia G, Silvestri S, Carrassa L, et al: A unique pattern of ET-743 activity in different cellular systems with defined deficiencies in DNA-repair pathways. Int J Cancer 92: 583-588, 2001.

8. Kanzaki A, Takebayashi Y, Ren XQ, et al: Overcoming multidrug drug resistance in P-glycoprotein/MDR 1overexpressing cell lines by ecteinascidin 743. Mol Cancer Ther 1: 1327-1334, 2002.

9. Delaloge S, Yovine A, Taamma A, Riofrio M, Brain E, Raymond E, Cottu P, Goldwasser F, Jimeno J, Misset JL, Marty M and Cvitkovic E: Ecteinascidin-743: a marine-derived compound in advanced, pretreated sarcoma patients - preliminary evidence of activity. J Clin Oncol 19: 1248-1255, 2001.

10. Yovine A, Riofrio M, Blay JY, et al: Phase II study of ecteinascidin-743 in advanced pretreated soft tissue sarcoma patients. J Clin Oncol 22: 890-899, 2004.
11. Le Cesne A, Blay JY, Judson I, Van Oosterom A, Verweij J, Radford J, Lorigan P, Rodenhuis S, Ray-Coquard I, Bonvalot S, Collin F, Jimeno J, Di Paola E, Van Glabbeke M and Nielsen OS: Phase II study of ET-743 in advanced soft tissue sarcomas: a European Organisation for the Research and Treatment of Cancer (EORTC) soft tissue and bone sarcoma group trial. J Clin Oncol 23: 576-584, 2005.

12. Garcia-Carbonero R, Supko JG, Manola J, Seiden MV, Harmon D, Ryan DP, Quigley MT, Merriam P, Canniff J, Goss G, Matulonis U, Maki RG, Lopez T, Puchalski TA, Sancho MA, Gomez J, Guzman C, Jimeno J and Demetri GD: Phase II and pharmacokinetic study of ecteinascidin 743 in patients with progressive sarcomas of soft tissues refractory to chemotherapy. J Clin Oncol 22: 1480-1490, 2005.

13. Le Cesne A, Judson I, Crowther D, et al: Randomized phase III study comparing conventional-dose doxorubicin plus ifosfamide versus high-dose doxorubicin plus ifosfamide plus recombinant human granulocyte-macrophage colony-stimulating factor in advanced soft tissue sarcomas: a trial of the European Organisation for Research and Treatment of Cancer/Soft Tissue and Bone Sarcoma Group. J Clin Oncol 18: 2676-2684, 2002.

14. Demetri GD, Chawla SP, von Mehren M, Ritch P, Baker LH, Blay JY, Hande KR, Keohan ML, Samuels BL, Schuetze S, Lebedinsky C, Elsayed YA, Izquierdo MA, Gómez J, Park YC and Le Cesne A: Efficacy and safety of trabectedin in patients with advanced or metastatic liposarcoma or leiomyosarcoma after failure of prior anthracyclines and ifosfamide: results of a randomized phase II study of two different schedules. J Clin Oncol 27: 4188-4196, 2009.

15. Baruchel S, Pappo A, Krailo M, Baker KS, Wu B, Villaluna D, Lee-Scott M, Adamson PC and Blaney SM: A phase 2 trial of trabectedin in children with recurrent rhabdomyosarcoma, Ewing sarcoma and non-rhabdomyosarcoma soft tissue sarcomas: a report from the Children's Oncology Group. Eur J Cancer 48: 579-585, 2012.

16. Grosso F, Jones RL, Demetri GD, Judson IR, Blay JY, et al: Efficacy of trabectedin (ecteinascidin-743) in advanced pretreated myxoid liposarcomas: a retrospective study. Lancet Oncol 8: 595-602, 2007.

17. Fayette J, Boyle H, Chabaud S, et al: Efficacy of trabectedin for advanced sarcomas in clinical trials versus compassionate use programs: analysis of 92 patients treated in a single institution. Anticancer Drugs 21: 113-119, 2010.

18. Theman TA, Hartzell TL, Sinha I, et al: Recognition of a new chemotherapeutic vesicant: trabectedin (Ecteinascidin-743) extravasation with skin and soft tissue damage. J Clin Oncol 27: 198-200, 2009.

19. van Glabbeke M, Verweij J, Judson I, et al: EORTC Soft Tissue and Bone Sarcoma Group. Progression-free rate as the principal end-point for phase II trials in soft-tissue sarcomas. Eur J Cancer 38: 543-549, 2002 